\title{
Preface: Joint Discussion JD4 Ultraviolet emission in early-type galaxies
}

The presence of enhanced ultraviolet (UV) emission in early-type galaxies, which are dominated by old stellar populations, has been a puzzle for more than 40 years. The observed UV flux plausibly contains contributions from both evolved stars (traditionally referred to as the 'UV upturn' phenomenon) and young stars. While the UV upturn is well-characterised observationally, a firm understanding of its origin still eludes us. Similarly, while recent results from GALEX and deep optical surveys strongly indicate the presence of small mass fractions of young stars across low and intermediate redshift, the exact mechanism (e.g. stellar mass loss, minor mergers) that drives this star formation is not fully understood. The role of active galactic nuclei in regulating the low-level star formation at late epochs also remains relatively unexplored in galaxy formation models.

This joint discussion meeting aimed to deliver a review of each of the topics outlined above. It discussed the observational evidence for the UV-upturn, for example its correlation with other bulk galaxy properties such as metallicity, and to explore the various theories of its origin. Primarily, these are single-star channels (e.g. stars on the extreme horizontal branch and the post-asymptotic giant branch) and binary stars, both with extended distributions.

New UV results that demonstrate the presence of small mass fractions of young stars in early-type galaxies were explored, especially in the context of the principal driver of this low-level star formation. Traditional recipes for AGN feedback, which typically truncate star formation in massive galaxies at early epochs, must be revisited to accomodate the low levels of star formation observed in nearby early-type galaxies. The meeting reviewed the role of AGN feedback in the evolution of early-type galaxies and examined how the most recent UV results can further constrain the feedback recipes employed in the models.

With the recently renewed capability of HST (via the Wide Field Camera 3) to image the UV with a high spatial resolution and a wide field-of-view, many exciting results are expected over the next few years. For example, recent star formation should result in UV 'fine structure' on small spatial scales, as opposed to the smooth light profile expected from the UV upturn (since it is produced by the old, underlying stellar population). With the advent of the JWST and the extremely large telescopes, it will be possible to trace rest-frame UV emission in early-type galaxies both to unprecedentedly high redshift and at higher spatial resolution than is possible with current instruments. This meeting aimed to revisit the old issue of UV emission in early-type galaxies in a new light, with a view to preparing the ground for more definitive studies with forthcoming instrumentation.

Sugata Kaviraj

SOC Chair JD4 